\title{
Thyroid hormone signaling specifies cone subtypes in human retinal organoids
}

Kiara C. Eldred ${ }^{1}$, Sarah E. Hadyniak ${ }^{1}$, Katarzyna A. Hussey ${ }^{1}$, Boris Brennerman ${ }^{1}$, Pingwu Zhang ${ }^{2}$, Xitiz Chamling ${ }^{2}$, Valentin M. Sluch ${ }^{3}$, Derek S. Welsbie ${ }^{4}$, Samer Hattar $^{5}$, James Taylor ${ }^{1}$, Karl Wahlin ${ }^{4}$, Donald J. Zack ${ }^{2,6}$, and Robert J. Johnston Jr. ${ }^{1 *}$

${ }^{1}$ Department of Biology, Johns Hopkins University, 3400 N. Charles Street, Baltimore, MD 21218, United States

${ }^{2}$ Wilmer Eye Institute, Johns Hopkins University School of Medicine, Baltimore, MD 21287, United States

${ }^{3}$ Department of Ophthalmology, Novartis Institutes for BioMedical Research, Cambridge, Massachusetts, United States

${ }^{4}$ Shiley Eye Institute, University of California San Diego, La Jolla, CA, United States

${ }^{5}$ National Institute of Mental Health, National Institutes of Health, Bethesda, MD 20892, United States

${ }^{6}$ Departments of Molecular Biology and Genetics, Neuroscience, and Institute of Genetic Medicine, Johns Hopkins University School of Medicine, Baltimore, MD 21287, United States

* Correspondence to: robertjohnston@jhu.edu

One sentence summary: Cone specification in human organoids 


\begin{abstract}
The mechanisms underlying the specification of diverse neuronal subtypes within the human nervous system are largely unknown. The blue (shortwavelength/S), green (medium-wavelength/M) and red (long-wavelength/L) cone photoreceptors of the human retina enable high-acuity daytime vision and trichromatic color perception. Cone subtypes are specified in a poorly understood two-step process, with a first decision between $\mathrm{S}$ and L/M fates, followed by a decision between $\mathrm{L}$ and $\mathrm{M}$ fates. To determine the mechanism controlling $\mathrm{S}$ vs. L/M fates, we studied the differentiation of human retinal organoids. We found that human organoids and retinas have similar distributions, gene expression profiles, and morphologies of cone subtypes. We found that $\mathrm{S}$ cones are specified first, followed by L/M cones, and that thyroid hormone signaling is necessary and sufficient for this temporal switch. Temporally dynamic expression of thyroid hormone degrading and activating proteins supports a model in which the retina itself controls thyroid hormone levels, ensuring low signaling early to specify $\mathrm{S}$ cones and high signaling late to produce L/M cones. This work establishes organoids as a model for determining the mechanisms of cell fate specification during human development.
\end{abstract}

Key words: human retina, organoid, photoreceptor, cone cell, opsin, thyroid hormone receptor beta, Thrß, T3, T4, CRISPR/Cas9, DIO2, DIO3 
Cone photoreceptors in the human retina enable daytime, color, and high acuity vision (1). The three subtypes of human cones are defined by the visual pigment that they express: blue- (short-wavelength/S), green- (mediumwavelength/M), or red- (long-wavelength/L) opsin (2). Specification of human cones occurs in a two-step process. First, a decision occurs between S vs. L/M cone fates (Fig. 1A). If the L/M fate is chosen, a subsequent choice is made between expression of L- or M-opsins (3-6). Mutations affecting opsin expression or function cause various forms of color blindness and retinal degeneration (7-9). Great progress has been made in our understanding of the vertebrate eye through the study of model organisms. However, little is known about the developmental mechanisms that generate the mosaic of mutually exclusive cone subtypes in the human retina. Here, we study the specification of human cone subtypes using human retinal organoids differentiated from stem cells (Fig. 1DK).

Human retinal organoids generate photoreceptors that respond to light $(10-14)$. We find that human organoids recapitulate the specification of cone subtypes observed in the human retina, including the temporal generation of $S$ cones followed by $L$ and $M$ cones. Moreover, we find that this regulation is controlled by thyroid hormone signaling, which is necessary and sufficient to control cone subtype fates through the nuclear hormone receptor Thyroid Hormone Receptor $\beta$ (Thr $\beta$ ). Expression of thyroid hormone-regulating genes suggests that retina-intrinsic temporal control of thyroid hormone levels and activity governs cone subtype specification. While retinal organoids have largely 
been studied for their promise of therapeutic applications (15), our work demonstrates that human organoids can also be used to reveal fundamental mechanisms of human development.

\section{Specification of cone cells in organoids recapitulates development in the}

\section{human retina}

We compared features of cone subtypes in human organoids to adult retinal tissue. Adult human retinas and organoids at day 200 of differentiation displayed similar ratios of $\mathrm{S}$ to $\mathrm{L} / \mathrm{M}$ cones as indicated by expression of $\mathrm{S}$ - or L/M-opsins (adult: S=13\%, L/M=87\%; organoid: S=29\%, L/M=71\%)(Fig. 1B-C, S1A). We examined L/M cones with an antibody that recognizes both $L-$ and $M-$ opsin proteins due to their extremely high similarity. Both $S$ and L/M cones expressed the cone-rod-homeobox transcription factor (CRX), a critical transcription factor for photoreceptor differentiation (Fig. 2A, E)(16-18), indicating proper fate specification in organoids. Additionally, cones in organoids and retinas displayed similar morphologies, with L/M cones that had longer outer segments and wider inner segments than S cones (Fig. 2B-D, F-H)(19). The outer segments of cones were shorter in organoids than in adult retinas, consistent with postnatal maturation (Fig. 2D, H)(20). Thus, cone subtypes in human retinal organoids displayed distributions, gene expression patterns, and morphologies similar to cones of the human retina.

We next examined the developmental dynamics of cone subtype specification in organoids. In the human retina, S cones are generated during 
fetal weeks 11-34 (days 77-238), whereas LM cones are specified later during fetal weeks 14-37 (days 98-259)(21, 22). We tracked the ratios and densities of S and L/M cones in organoids by antibody staining over 360 days of differentiation. A significant number of cones expressing S-opsin were first observed at day 150 (Fig. 2I, L-M). The density of S cones leveled off at day 170 (Fig. 2M), at the timepoint when cones expressing L/M-opsin began to be observed (Fig. 2J-M). The population of L/M cones increased dramatically until day 300 (Fig. 2K-M) when they reached a steady-state density. Remarkably, the 20-day difference between S- and L/M-opsin expression onset in retinal organoids is similar to the 20-day difference observed in the appearance of S- and L/M-cones in the fetal retina (21). These observations show a temporal switch from $\mathrm{S}$ cone specification to L/M cone specification during retinal development.

We next conducted RNA-Seq through 250 days of iPSC-derived organoid development. We found that S-opsin RNA was expressed first at day 111 and leveled off at day 160, while L/M-opsin RNA was expressed at day 160 and remained steady after day 180 , consistent with the timeline of photoreceptor maturation in organoids and fetal retinas (Fig. 2N, Fig S1B). Moreover, CRX RNA and CRX protein were expressed before opsins in organoids, similar to human development (23) (Fig. 2N, Fig. S1B-G,). Thus, human organoids recapitulate many aspects of the developmental timeline of cone subtype specification observed in human retinas, providing a model system to uncover the mechanisms of these developmental changes. 


\section{Thyroid hormone signaling is necessary and sufficient for the temporal switch between $S$ and $L / M$ fate specification}

Seminal work in mice identified thyroid hormone receptor $\beta 2$ (Thr $\beta 2)$ as a critical regulator of cone subtype specification: Thrß2 mutants display a complete loss of M-opsin expression and a complete gain of S-opsin expression in cone photoreceptors (24-26). Similar roles for Thrß2 have been characterized in other

organisms with highly divergent cone patterning (27-29). Additionally, rare human mutations in Thrß2 are reported to alter color perception, indicative of a change in the $S$ to $L / M$ cone ratio (30). To directly test the role of Thrß2 in human cone subtype specification, we used CRISPR/Cas9 in human embryonic stem cells (ESCs) to generate a homozygous mutation resulting in early translational termination in the unique first exon of Thrß2 (Fig. S2A). Surprisingly, organoids derived from these mutant stem cells displayed no changes in cone subtype ratio or density (wild-type: $\mathrm{S}=62 \%$, L/M=38\%; Thrß2 KO: S=59\%, L/M=41\%; P=0.83), indicating that, unlike previous suggestions based on other species, Thr $\beta 2$ is dispensable for cone subtype specification in humans (Fig. 3A-C).

Since Thrß2 alone is not required for human cone subtype specification, we reexamined data from Weiss et. al (30) and found that missense mutations in exons 9 and 10 affected both Thrß2 and another isoform of the human Thr $\beta$ gene, Thr $\beta 1$ (Fig. S2A). Thus, we asked whether Thr $\beta 1$ and Thr $\beta 2$ together are required for cone subtype specification in humans. To completely ablate Thr $\beta$ function (i.e. Thr $\beta 1$ and Thr $\beta 2$ ), we used CRISPR/Cas9 in human ESCs to delete a shared exon that codes for part of the DNA-binding domain (DBD) of Thr $\beta$ (Fig. 
S2A). Thr $\beta$ null mutant retinal organoids displayed a complete conversion of all cones to the S subtype (wild-type: $S=27 \%$, L/M=73\%; Thr $\beta$ KO: S=100\%, $\mathrm{L} / \mathrm{M}=0 \%$; $\mathrm{P}<0.0001$ ) (Fig. 3D-E, H). In these mutants, all cones expressed Sopsin and had the $S$ cone morphology (Fig. 3I-J). Thus, Thr $\beta$ is required to activate $\mathrm{L} / \mathrm{M}$ and to repress $\mathrm{S}$ cone fates in the human retina.

Thr $\beta$ binds with high affinity to triiodothyronine (T3), the more active form of thyroid hormone, to regulate gene expression (31). Since L/M cones differentiate after $S$ cones, we hypothesized that $T 3$ acts through $\operatorname{Thr} \beta$ late in retinal development to induce $\mathrm{L} / \mathrm{M}$ cone fate and repress $\mathrm{S}$ cone fate. One prediction of this hypothesis is that addition of T3 early in development will induce L/M fate and repress S fate. To test this model, we added 20nM T3 to ESC- and iPSC-derived organoids starting from days 20-50 to day 200 of differentiation. We observed a dramatic conversion of cone cells to L/M fate (wild-type: S=27\%, L/M=73\%; wild-type + T3: S=4\%, L/M=96\%; P<0.01) (Fig. 3F, H, Fig. S2B). Thus, early addition of T3 is sufficient to induce L/M fate and suppress $\mathrm{S}$ fate.

To test whether T3 acts specifically through $\operatorname{Thr} \beta$ to control cone subtype specification, we differentiated Thr $\beta$ mutant organoids with early T3 addition. Thr $\beta$ mutation completely suppressed the effects of $\mathrm{T} 3$, generating organoids with only S cones (wild-type + T3: S=4\%, L/M=96\%; Thr $\beta$ KO + T3: S=100\%, $L / M=0 \% ; P<0.0001$ ) (Fig. 3F-H). We conclude that $T 3$ acts though $T h r \beta$ to promote L/M cone fate and suppress $\mathrm{S}$ cone fate. 
We confirmed the regulation of L/M-opsin expression through thyroid hormone signaling in a retinoblastoma cell line, which expresses L/M-opsin when treated with T3 (Fig. S2C-D)(32). T3-induced activation of L/M-opsin expression was suppressed upon RNAi knock down of Thr $\beta$ (Fig. S2E-F), similar to the suppression observed in human organoids.

Interestingly, in organoids, early T3 addition not only converted cone cells to L/M fate but also dramatically increased cone density (Fig. 3F, K). Moreover, T3 acts specifically through $\operatorname{Thr} \beta$ to control cone density (Fig. 3G, K). Early T3 addition may increase cone density by advancing and extending the temporal window of L/M cone generation.

Together, these results demonstrate that T3 signals though Thr $\beta$ to promote $\mathrm{L} / \mathrm{M}$ cone fate and repress $\mathrm{S}$ cone fate in developing human retinal tissue.

\section{Dynamic expression of thyroid hormone-regulating genes during}

\section{development}

Our data suggest that temporal control of thyroid hormone signaling determines the S vs. L/M cone fate decision, whereby low signaling early induces $S$ fate and high signaling late induces L/M fate. Thyroid hormone exists largely in two states: Thyroxine (T4), the most abundant circulating form of thyroid hormone, and T3, which binds thyroid hormone receptors with high affinity (31, 33). Conversion of T4 to T3 occurs locally in target tissues to induce gene expression responses $(34,35)$. Deiodinases, enzymes that modulate the levels 
of T3 and T4, are expressed in the retinas of mice, fish, and chicken $(29,36-40)$. Therefore, we predicted that T3- and T4-degrading enzymes would be expressed during early human eye development to reduce thyroid hormone signaling and specify S cones, while T3-producing enzymes, carriers, and transporters would be expressed later in human eye development to increase signaling and generate L/M cones.

To test these predictions, we examined gene expression across 250 days of organoid development. The expression patterns of thyroid hormone-regulating genes were grouped into three classes: changing expression (Fig. 4A), consistent expression (Fig. 4B), or no expression (Fig. 4C). Interestingly, Deiodinase 3 (DIO3), an enzyme that degrades T3 and T4 (34), was expressed at high levels early in organoid development but at low levels later (Fig. 4A). Conversely, Deiodinase 2 (DIO2), an enzyme that converts T4 to active T3 (34), was expressed at low levels early but then dramatically increased over time (Fig. 4A). We examined RNA-Seq data from Hoshino et. al (23) and found that developing human retinas display similar temporal changes in expression of DIO3 and DIO2 (Fig. S3A). Deiodinase 1 (DIO1), which regulates T3 and T4 predominantly in the liver and kidney (41), was not expressed in organoids or retinas (Fig. 4C, S3C). Thus, the dynamic expression of Dio3 and Dio2 supports low thyroid hormone signaling early in development to generate S cones and high thyroid hormone signaling late to produce L/M cones.

Consistent with a role for high thyroid hormone signaling in the generation of L/M cones later in development, expression of transthyretin (TTR), a thyroid 
hormone carrier protein, increased during organoid and retinal development (Fig.

4A, S3A)(23). In contrast, albumin $(A L B)$ and thyroxine-binding globulin (SERPINA7), other carrier proteins of T3 and T4, were not expressed in organoids or retinas (Fig. 4C, S3C)(23).

T3 and T4 are transported into cells via membrane transport proteins (42). The T3/T4 transporters SLC7A5 and SLC7A8 increased in expression during organoid differentiation (Fig. 4A). Additionally, two T3/T4 transporters, SLC3A2 and SLC16A2, were expressed at high and consistent levels throughout organoid development (Fig. 4B). Other T3/T4 transporters (SLC16A10, SLCO1C1, SLC5A5) were not expressed in organoids (Fig. 4C), suggesting tissue-specific regulation of T3/T4 uptake. We observed similar expression patterns of T3/T4 transporters in human retinas (Fig. S3A-C)(23).

We next examined expression of transcriptional activators and repressors that mediate the response to thyroid hormone. Consistent with Thr $\beta$ expression in human cones (43), expression of $T h r \beta$ in organoids increased with time as cone cells were specified (Fig. 4A). Expression of thyroid hormone receptor $\alpha$ (Thr $\alpha$ ) similarly increased with time (Fig. 4A). Thyroid hormone receptor cofactors, co-repressor NCoR2 and co-activator MED1, were expressed at steady levels during organoid differentiation (Fig. 4B). Similar temporal expression patterns were observed in human retinas (Fig. S3A-B)(23). Thus, our data suggest that expression of Thr $\beta$ and other transcriptional regulators enables gene regulatory responses to differential thyroid hormone levels. 
A complex pathway controls production of thyroid hormone. Thyrotropinreleasing hormone $(\mathrm{TRH})$ is produced by the hypothalamus and other neural tissue. TRH stimulates release of thyroid-stimulating hormone $\alpha$ (CGA) and thyroid-stimulating hormone $\beta$ (TSH $\beta$ ) from the pituitary gland. CGA and TSH $\beta$ bind the thyroid-stimulating hormone receptor (TSHR) in the thyroid gland. T3 and T4 production requires Thyroglobulin (TG), the substrate for T3/T4 synthesis, and Thyroid Peroxidase (TPO), an enzyme which iodinates tyrosine residues in TG (44). Interestingly, TRH was expressed in organoids and retinas but the other players were not (Fig. 4A-C, S3A-C) $(23,45,46)$, suggesting that the retina itself does not generate thyroid hormone, rather it modulates the relative levels of T3 and T4 and expresses TRH to signal for thyroid hormone production in other tissues.

Therefore, the temporal expression of thyroid hormone signaling regulators supports our model that the retina intrinsically controls T3 and T4 levels, ensuring low thyroid hormone signaling early to promote $S$ fate and high thyroid hormone signaling late to specify L/M fate (Fig. 4D).

Organoids provide a powerful system to determine the mechanisms of human development. Model organism and epidemiological studies generate important hypotheses about human biology that are often experimentally intractable. This work shows that organoids enable direct testing of hypotheses in developing human tissue. 
Our studies identify temporal regulation of thyroid hormone signaling as a mechanism controlling cone subtype specification in humans. Consistent with our findings, preterm human infants with low T3/T4 have an increased incidence of color vision defects (47-50). Moreover, our identification of a mechanism that generates one cone subtype while suppressing the other is critical for developing organoid-based therapies to treat diseases such as color blindness, retinitis pigmentosa, and macular degeneration. 


\section{Figure Legends:}

Figure 1. $S$ and L/M cone generation in human retinal organoids

A) Decision between $S$ and L/M cone subtype fate.

B-C) S-opsin (blue) L/M-opsin (green).

B) Human adult retina age 53.

C) iPSC-derived organoid, day 200 of differentiation.

D-K) Bright field images of organoids derived from iPSCs.

D) Undifferentiated iPSCs.

E) Day 1: aggregation.

F) Day 4: formation of neuronal vesicles.

G) Day 8: differentiation of retinal vesicles.

H) Day 12: manual isolation of retinal organoid.

I) Day 43: arrow indicates developing retinal tissue, arrowhead indicates developing retinal pigment epithelium (RPE).

J) Day 199: arrow indicates outer segments.

K) Day 330: arrow indicates outer segments.

Figure 2. Human cone subtype specification is recapitulated in organoids A-K) S-opsin (blue) and L/M-opsin (green) were examined in human iPSCderived organoids (2A, C-E, G-M) and human retinas (2B, D, F, H). A-C, E-G) Arrows indicate outer segments, full arrowheads indicate inner segments, empty arrowheads indicate nuclei. 
$A, E) C R X$ (a general marker of photoreceptors) is expressed in $S$ cones and L/M cones.

B-D) S cones display short outer segments and thin inner segments in both human retinas and organoids.

F-H) L/M cones display long outer segments and wide inner segments in both human retinas and organoids.

D,H) Quantification of outer segment lengths and inner segment widths (adult retina: $L / M, n=13, S, n=10$; organoid: $L / M, n=35, S, n=42)$.

I-N) S cones are generated before L/M cones in organoids.

L) Ratio of S:L/M cones during organoid development.

M) Density of S and L/M cones during organoid development.

N) S-opsin expression precedes L/M-opsin expression in human iPSC-derived organoids. $C R X$ expression starts before opsin expression. TPM=Transcripts per Kilobase Million.

Figure 3. Thyroid hormone signaling is necessary and sufficient for the temporal switch between S and L/M fate specification

A-K) S-opsin (blue) and L/M-opsin (green) were examined in human ESCderived organoids.
A) Wild-type (WT)
B) Thrß2 early termination mutant (Thrß2 KO).
C) Quantification of A-B (WT n=3, Thrß2 KO n=3)
D) Wild-type (WT) 
E) Thr K Knockout ( $T h r \beta \mathrm{KO})$

F) WT treated with $20 \mathrm{nM} \mathrm{T3}(\mathrm{WT}+\mathrm{T} 3)$.

G) Thr $\beta$ KO treated with $20 \mathrm{nM} \mathrm{T3}(\operatorname{Thr} \beta \mathrm{KO}+\mathrm{T} 3)$.

H) Quantification of D-E (WT, n=9; Thr $\beta$ KO, n=3; WT + T3, n=6; Thr $\beta$ KO + T3, $\mathrm{n}=3$. Tukey's multiple comparisons test comparisons test: WT vs Thr $\beta$ KO, $\mathrm{P}<0.0001 ; \mathrm{WT}$ vs WT + T3, $\mathrm{P}<0.01 ; \mathrm{WT}+\mathrm{T} 3$ vs Thr $\beta$ KO + T3, P<0.0001). I) Length of outer segments (WT, L/M n=66 cells, WT, S n=66 cells, Thr $\beta$ KO, $\mathrm{n}=50$ cells. Tukey's multiple comparisons test: WT L/M vs. WT SW, $\mathrm{P}<0.0001$; WT L/M vs. Thr $\beta$ KO, P<0.0001; WT S vs. Thr $\beta$ KO, not significantly different). J) Width of inner segments (WT, L/M n=78 cells; WT, S n=78 cells; Thr $\beta$ KO, $\mathrm{n}=118$ cells. Tukey's multiple comparisons test: WT L/M vs. WT SW, $\mathrm{P}<0.0001$; WT L/M vs. Thr $\beta$ KO, P<0.0001; WT S vs. Thr $\beta$ KO, not significantly different). K) T3 acts through Thr $\beta$ to increase total cone number. Quantification of density of $S$ and L/M cones. (WT, n=6; Thr $\beta$ KO, n=3; WT + T3, n=3; Thr $\beta$ KO + T3, n=3. Tukey multiple comparisons test between total cone numbers: WT vs. Thr $\beta$ KO, not significantly different; WT vs WT + T3, P<0.01; WT + T3 vs Thr $\mathrm{KO}+\mathrm{T} 3$, $\mathrm{P}<0.0001)$.

Figure 4. Dynamic expression of thyroid hormone signaling regulators during development

A-C) Heat maps of Log(Transcripts per Kilobase Million (TPM) + 1) values for genes with (A) changing expression, (B) consistent expression, and (C) no expression. Numbers at the bottom of heat maps indicate organoid age in days. 
D) Model of the temporal mechanism of cone subtype specification in humans.

For simplicity, only the roles of DIO3 and DIO2 are illustrated. In step 1,

expression of DIO3 degrades T3 and T4 leading to S cone specification. In step

2, expression of DIO2 converts $\mathrm{T} 4$ to $\mathrm{T} 3$ to signal $\mathrm{Thr} \beta$ to repress $\mathrm{S}$ and induce

L/M cone fate. 


\section{References:}

1. K. Viets, K. C. Eldred, R. J. Johnston, Jr., Mechanisms of Photoreceptor Patterning in Vertebrates and Invertebrates. Trends in genetics : TIG, (2016).

2. J. Nathans, D. Thomas, D. Hogness, Molecular Genetics of Human Color Vision: The Genes Encoding Blue, Green, and Red Pigments. Science 232, 193-202 (1986).

3. D. Vollrath, J. Nathans, R. W. Davis, Tandem array of human visual pigment genes at Xq28. Science 240, 1669-1672 (1988).

4. Y. Wang et al., A locus control region adjacent to the human red and green visual pigment genes. Neuron 9, 429-440 (1992).

5. P. M. Smallwood, Y. Wang, J. Nathans, Role of a locus control region in the mutually exclusive expression of human red and green cone pigment genes. Proceedings of the National Academy of Sciences of the United States of America 99, 1008-1011 (2002).

6. Y. Wang et al., Mutually exclusive expression of human red and green visual pigment-reporter transgenes occurs at high frequency in murine cone photoreceptors. Proceedings of the National Academy of Sciences of the United States of America 96, 5251-5256 (1999).

7. J. Nathans et al., Molecular genetics of human blue cone monochromacy. Science 245, 831-838 (1989). 
8. A. S. Ladekjaer-Mikkelsen, T. Rosenberg, A. L. Jorgensen, A new mechanism in blue cone monochromatism. Hum Genet 98, 403-408 (1996).

9. E. J. Patterson et al., Cone Photoreceptor Structure in Patients With XLinked Cone Dysfunction and Red-Green Color Vision Deficiency. Investigative ophthalmology \& visual science 57, 3853-3863 (2016).

10. T. Nakano et al., Self-formation of optic cups and storable stratified neural retina from human ESCs. Cell stem cell 10, 771-785 (2012).

11. X. Zhong et al., Generation of three-dimensional retinal tissue with functional photoreceptors from human iPSCs. Nature communications $\mathbf{5}$, 4047 (2014).

12. K. J. Wahlin et al., Photoreceptor Outer Segment-like Structures in LongTerm 3D Retinas from Human Pluripotent Stem Cells. Sci Rep 7, 766 (2017).

13. R. Kaewkhaw et al., Transcriptome Dynamics of Developing Photoreceptors in Three-Dimensional Retina Cultures Recapitulates Temporal Sequence of Human Cone and Rod Differentiation Revealing Cell Surface Markers and Gene Networks. Stem cells (Dayton, Ohio) 33, 3504-3518 (2015).

14. M. J. Phillips et al., A Novel Approach to Single Cell RNA-Sequence Analysis Facilitates In Silico Gene Reporting of Human Pluripotent Stem Cell-Derived Retinal Cell Types. Stem cells (Dayton, Ohio) 36, 313-324 (2018). 
15. A. Artero Castro, D. Lukovic, P. Jendelova, S. Erceg, Concise Review: Human Induced Pluripotent Stem Cell Models of Retinitis Pigmentosa. Stem cells (Dayton, Ohio) 36, 474-481 (2018).

16. T. Furukawa, E. M. Morrow, C. L. Cepko, Crx, a novel otx-like homeobox gene, shows photoreceptor-specific expression and regulates photoreceptor differentiation. Cell 91, 531-541 (1997).

17. C. L. Freund et al., Cone-rod dystrophy due to mutations in a novel photoreceptor-specific homeobox gene (CRX) essential for maintenance of the photoreceptor. Cell 91, 543-553 (1997).

18. S. Chen et al., Crx, a novel Otx-like paired-homeodomain protein, binds to and transactivates photoreceptor cell-specific genes. Neuron 19, 10171030 (1997).

19. C. A. Curcio et al., Distribution and morphology of human cone photoreceptors stained with anti-blue opsin. The Journal of Comparative Neurology 312, 610-624 (1991).

20. A. Hendrickson, D. Drucker, The development of parafoveal and midperipheral human retina. Behav Brain Res 49, 21-31 (1992).

21. M. Xiao, A. Hendrickson, Spatial and temporal expression of short, long/medium, or both opsins in human fetal cones. The Journal of Comparative Neurology 425, 545-559 (2000).

22. C. A. Curcio, K. R. Sloan, R. E. Kalina, A. E. Hendrickson, Human photoreceptor topography. J Comp Neurol 292, 497-523 (1990). 
23. A. Hoshino et al., Molecular Anatomy of the Developing Human Retina. Developmental cell 43, 763-779 e764 (2017).

24. L. Ng et al., A thyroid hormone receptor that is required for the development of green cone photoreceptors. Nature genetics 27, 94-98 (2001).

25. M. R. Roberts, M. Srinivas, D. Forrest, G. Morreale de Escobar, T. A. Reh, Making the gradient: thyroid hormone regulates cone opsin expression in the developing mouse retina. Proceedings of the National Academy of Sciences of the United States of America 103, 6218-6223 (2006).

26. M. L. Applebury et al., Transient expression of thyroid hormone nuclear receptor TRbeta2 sets S opsin patterning during cone photoreceptor genesis. Developmental dynamics : an official publication of the American Association of Anatomists 236, 1203-1212 (2007).

27. S. C. Suzuki et al., Cone photoreceptor types in zebrafish are generated by symmetric terminal divisions of dedicated precursors. Proceedings of the National Academy of Sciences of the United States of America 110, 15109-15114 (2013).

28. M. Sjoberg, B. Vennstrom, D. Forrest, Thyroid hormone receptors in chick retinal development: differential expression of mRNAs for alpha and Nterminal variant beta receptors. Development 114, 39-47 (1992).

29. J. M. Trimarchi, S. Harpavat, N. A. Billings, C. L. Cepko, Thyroid hormone components are expressed in three sequential waves during development of the chick retina. BMC developmental biology 8, 101 (2008). 
30. A. H. Weiss, J. P. Kelly, D. Bisset, S. S. Deeb, Reduced L- and M- and increased S-cone functions in an infant with thyroid hormone resistance due to mutations in the THRbeta2 gene. Ophthalmic genetics 33, 187-195 (2012).

31. H. H. Samuels, J. S. Tsai, J. Casanova, F. Stanley, Thyroid hormone action: in vitro characterization of solubilized nuclear receptors from rat liver and cultured GH1 cells. The Journal of clinical investigation 54, 853865 (1974).

32. Y. Liu, L. Fu, D. G. Chen, S. S. Deeb, Identification of novel retinal target genes of thyroid hormone in the human WERI cells by expression microarray analysis. Vision research 47, 2314-2326 (2007).

33. A. Schroeder, R. Jimenez, B. Young, M. L. Privalsky, The ability of thyroid hormone receptors to sense 44 as an agonist depends on receptor isoform and on cellular cofactors. Molecular endocrinology 28, 745-757 (2014).

34. M. Dentice, A. Marsili, A. Zavacki, P. R. Larsen, D. Salvatore, The deiodinases and the control of intracellular thyroid hormone signaling during cellular differentiation. Biochimica et biophysica acta 1830, 39373945 (2013).

35. V. M. Darras, A. M. Houbrechts, S. L. Van Herck, Intracellular thyroid hormone metabolism as a local regulator of nuclear thyroid hormone receptor-mediated impact on vertebrate development. Biochimica et biophysica acta 1849, 130-141 (2015). 
36. L. Ng et al., Type 3 deiodinase, a thyroid-hormone-inactivating enzyme, controls survival and maturation of cone photoreceptors. The Journal of neuroscience : the official journal of the Society for Neuroscience $\mathbf{3 0}$, 3347-3357 (2010).

37. P. J. Bonezzi, M. E. Stabio, J. M. Renna, The Development of MidWavelength Photoresponsivity in the Mouse Retina. Current eye research, $1-8$ (2018).

38. E. Bagci et al., Deiodinase knockdown during early zebrafish development affects growth, development, energy metabolism, motility and phototransduction. PloS one 10, e0123285 (2015).

39. C. Guo et al., Intrinsic expression of a multiexon type 3 deiodinase gene controls zebrafish embryo size. Endocrinology 155, 4069-4080 (2014).

40. S. L. Bruhn, C. L. Cepko, Development of the pattern of photoreceptors in the chick retina. The Journal of neuroscience : the official journal of the Society for Neuroscience 16, 1430-1439 (1996).

41. A. C. Bianco, D. Salvatore, B. Gereben, M. J. Berry, P. R. Larsen, Biochemistry, cellular and molecular biology, and physiological roles of the iodothyronine selenodeiodinases. Endocr Rev 23, 38-89 (2002).

42. D. S. Sharlin, T. J. Visser, D. Forrest, Developmental and cell-specific expression of thyroid hormone transporters in the mouse cochlea.

Endocrinology 152, 5053-5064 (2011).

43. T. C. Lee, D. Almeida, N. Claros, D. H. Abramson, D. Cobrinik, Cell cyclespecific and cell type-specific expression of $\mathrm{Rb}$ in the developing human 
retina. Investigative ophthalmology \& visual science 47, 5590-5598 (2006).

44. E. J. Barrett, in Medical Physiology, 2e Updated Edition, W. B. E. Boulpaep, Ed. (Elsevier, Inc., Philadelphia, PA, 2012), chap. 49.

45. S. R. Dubovy et al., Expression of hypothalamic neurohormones and their receptors in the human eye. Oncotarget 8, 66796-66814 (2017).

46. E. Martino et al., Thyrotropin-releasing hormone-like material in human retina. Journal of endocrinological investigation 3, 267-271 (1980).

47. J. Rovet, N. Simic, The role of transient hypothyroxinemia of prematurity in development of visual abilities. Semin Perinatol 32, 431-437 (2008).

48. N. Simic, C. Westall, E. V. Astzalos, J. Rovet, Visual abilities at 6 months in preterm infants: impact of thyroid hormone deficiency and neonatal medical morbidity. Thyroid 20, 309-315 (2010).

49. S. A. Yassin, A. J. Al-Dawood, W. M. Al-Zamil, M. A. Al-Ghamdi, Z. N. AlKhudairy, Comparative study of visual dysfunctions in 6-10-year-old very preterm- and full-term-born children. Int Ophthalmol, (2018).

50. H. J. Dowdeswell, A. M. Slater, J. Broomhall, J. Tripp, Visual deficits in children born at less than 32 weeks' gestation with and without major ocular pathology and cerebral damage. Br J Ophthalmol 79, 447-452 (1995).

51. N. S. Bhise, K. J. Wahlin, D. J. Zack, J. J. Green, Evaluating the potential of poly(beta-amino ester) nanoparticles for reprogramming human 
fibroblasts to become induced pluripotent stem cells. Int J Nanomedicine

8, 4641-4658 (2013).

52. V. M. Sluch et al., Enhanced Stem Cell Differentiation and

Immunopurification of Genome Engineered Human Retinal Ganglion

Cells. Stem Cells Transl Med 6, 1972-1986 (2017).

53. J. Harrow et al., GENCODE: the reference human genome annotation for The ENCODE Project. Genome research 22, 1760-1774 (2012). 

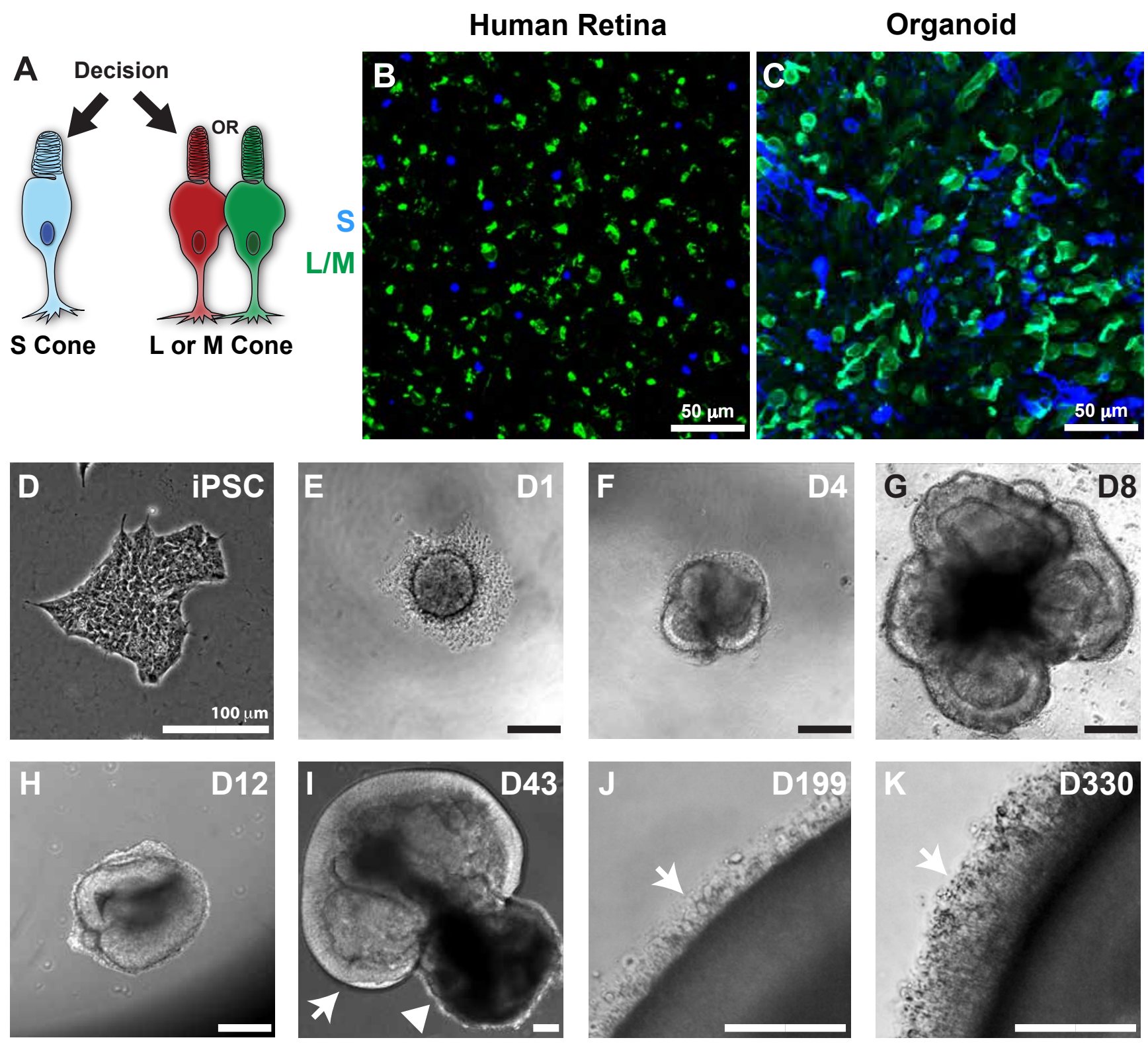

Figure 1 
bioRxiv preprint doi: https://doi.org/10.1101/359950; this version posted June 30, 2018. The copyright holder for this preprint (which was not certified by peer review) is the author/funder. All rights reserved. No reuse allowed without permission.
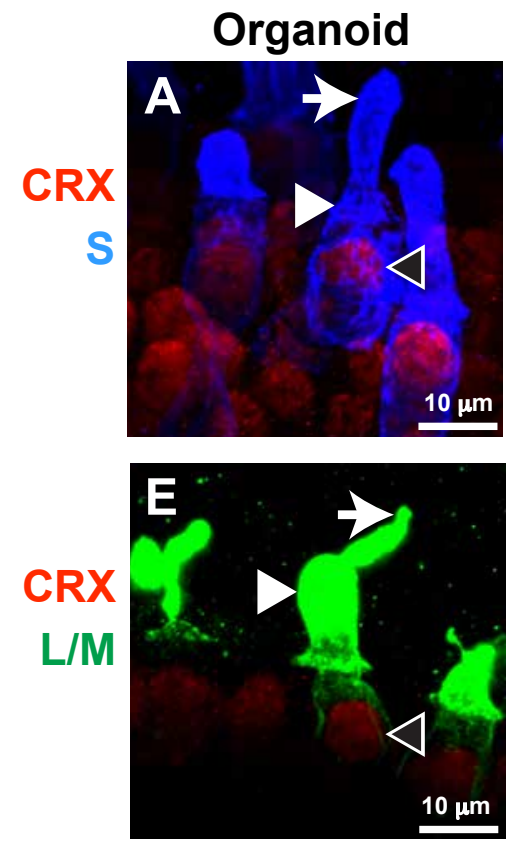

D150
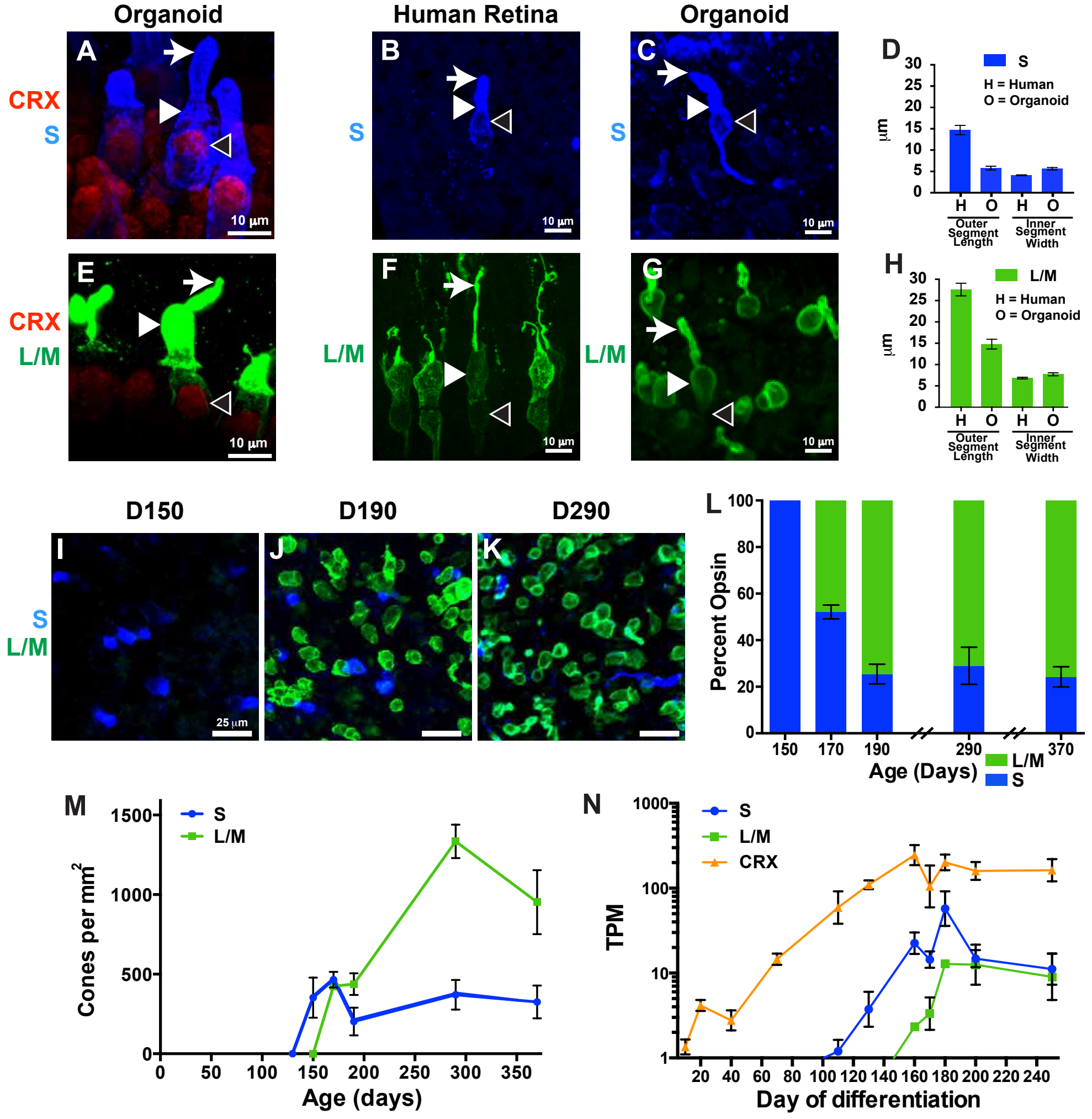

Figure 2 

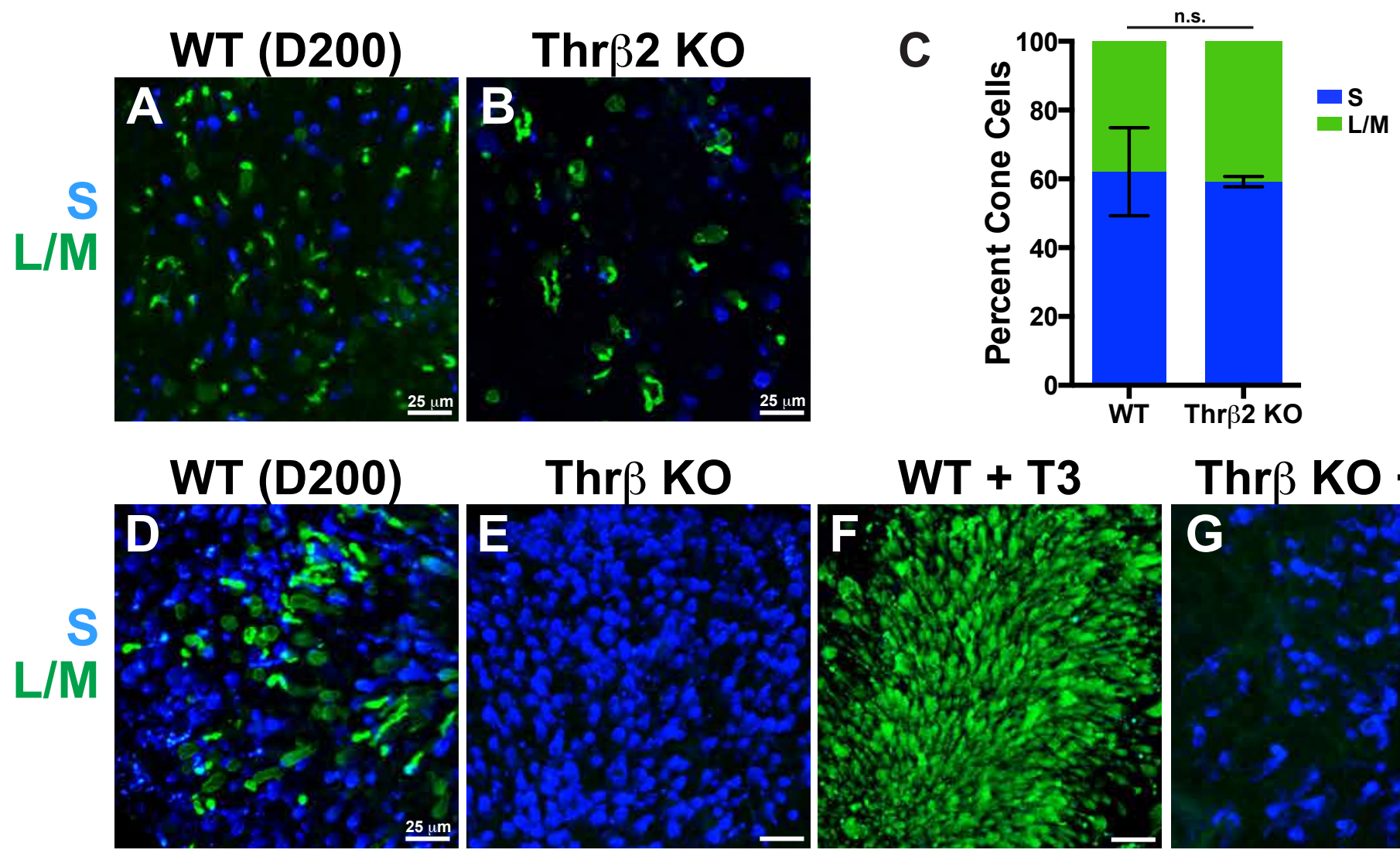

Thr $\beta \mathrm{KO}+\mathrm{T} 3$
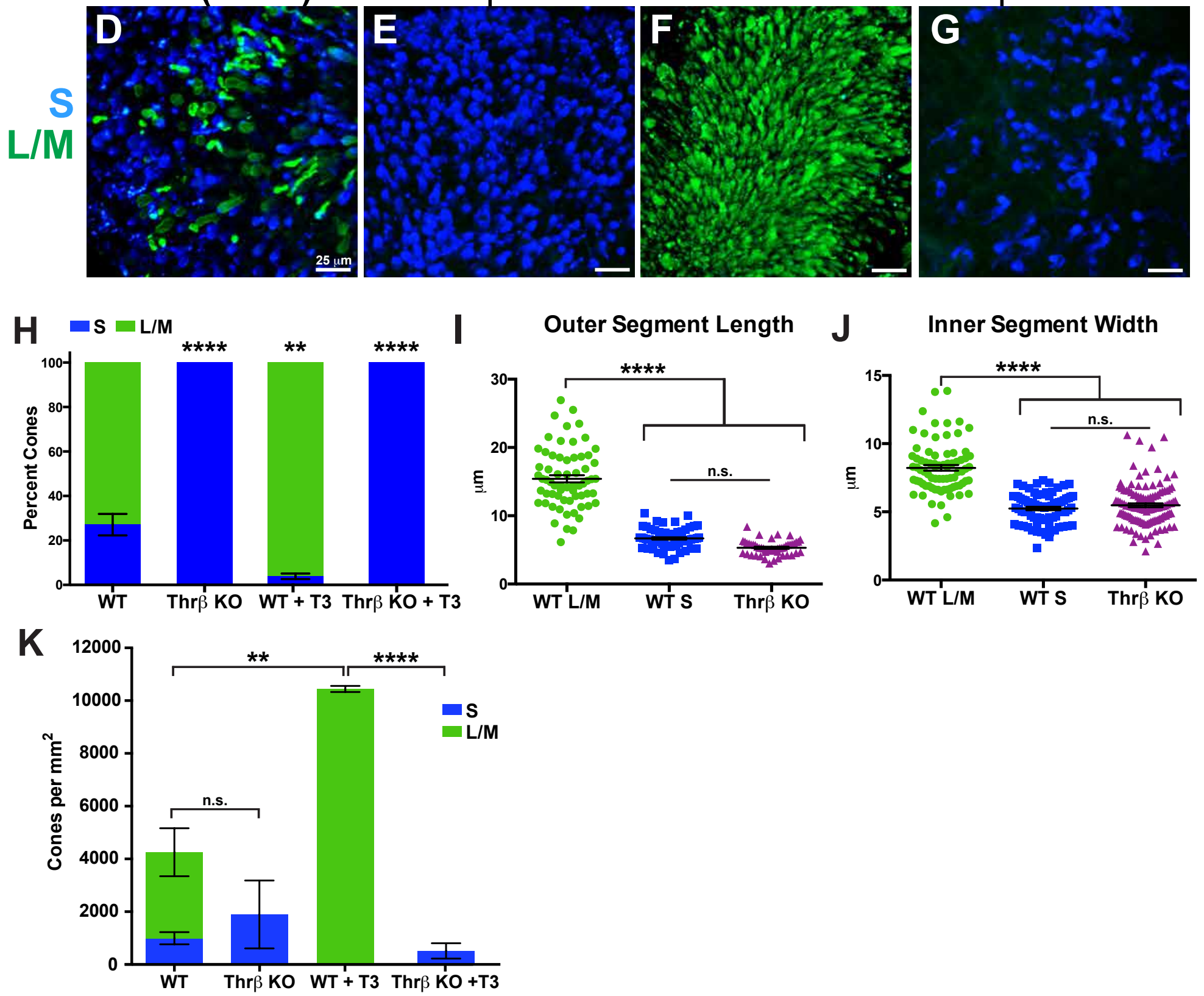

Figure 3 


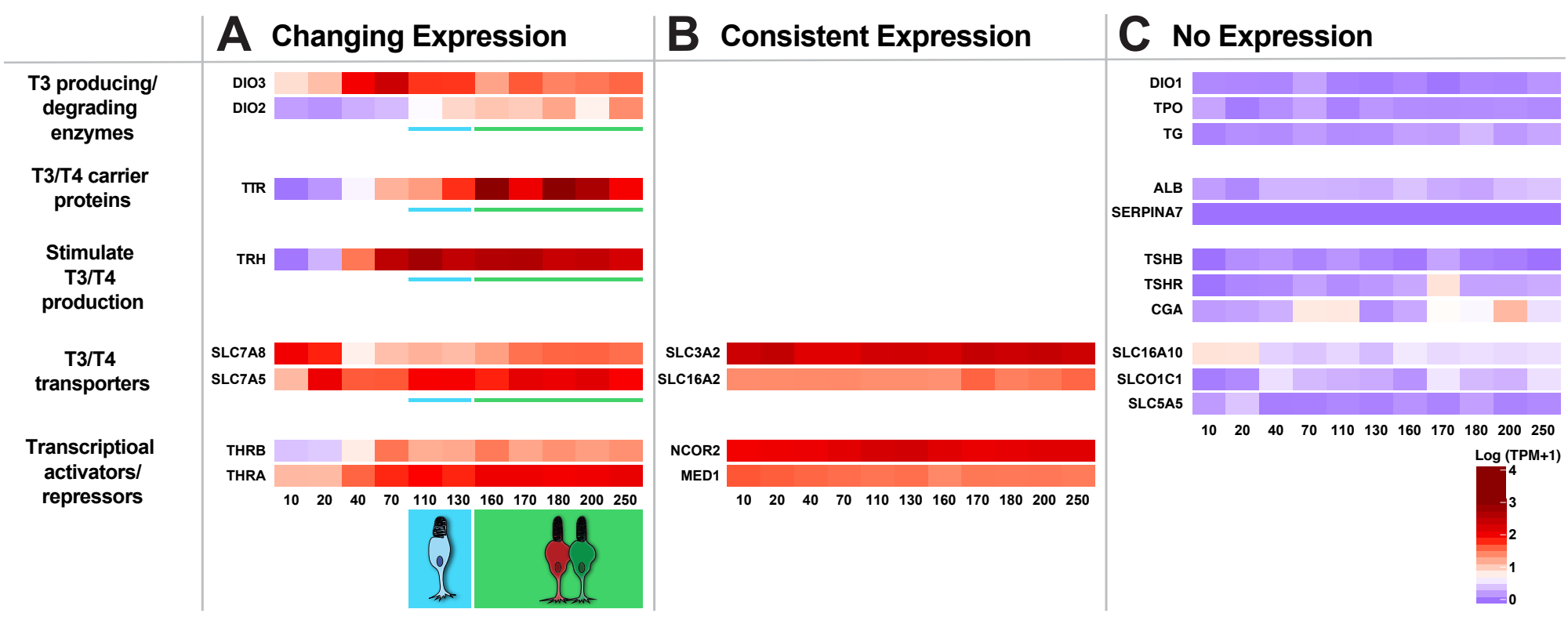

\section{Step 1: Low T3 thyroid hormone $\rightarrow \mathrm{S}$}

Step 2: High T3 thyroid hormone $\rightarrow$ L/M

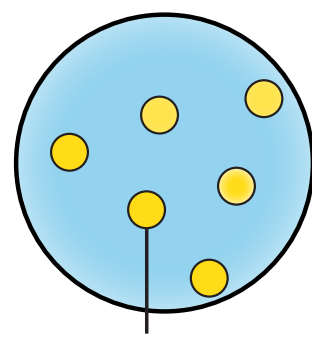

Naïve cone cell

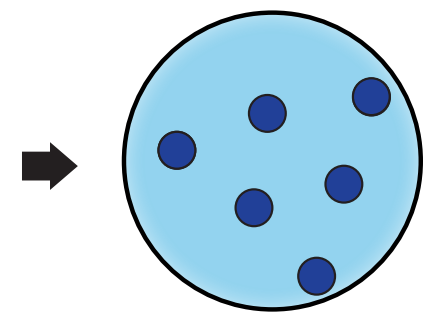

$\mathrm{DIO} 2$

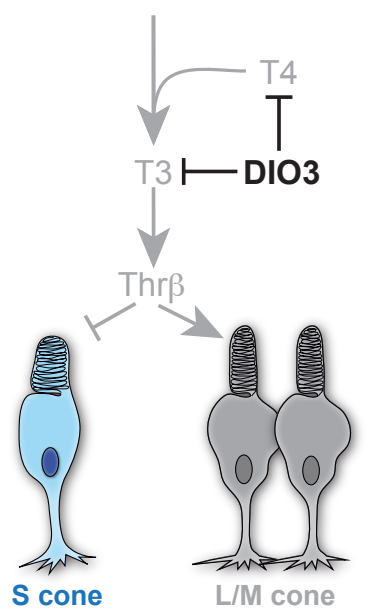

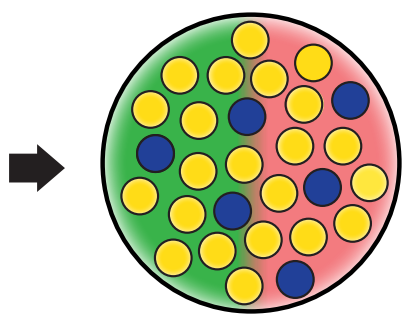
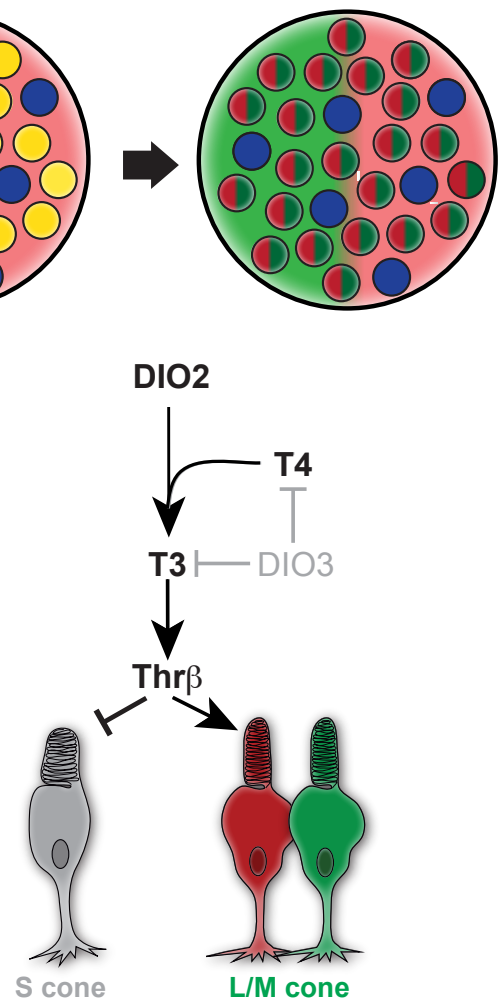

\section{Figure 4}

